\title{
TOWARDS THE BUILDING INFORMATION MODELING-BASED CAPITAL PROJECT LIFECYCLE MANAGEMENT IN THE LUXURY YACHT INDUSTRY
}

\author{
Fuyong Liu ${ }^{1}$ \\ Chaohe Chen ${ }^{2 *}$ \\ Wei $\mathbf{W u}^{3}$ \\ ${ }^{1}$ Department of Naval Architecture and Ocean Engineering, South China University of Technology, China \\ ${ }^{2}$ Department of Naval Architecture and Ocean Engineering, South China University of Technology, China \\ ${ }^{3}$ Department of Construction Management, California State University at Fresno, USA \\ * corresponding author
}

\begin{abstract}
It will be a new approach that BIM's capital project lifecycle management (CPLM) applied to the yacht industry. This paper explored the feasibility of applying the principles and rationales of BIM for capital project lifecycle management in luxury yacht design, engineering, fabrication, construction and operation. The paper examined the premises and backbone technology of BIM. It then evaluated leading naval engineering and shipbuilding software applications and their development trends from the functional lens of BIM. To systematically investigate a BIM-based approach for capital project lifecycle management (CPLM) in the luxury yacht industry, the paper proposed and outlined an implementation framework. A case study and a student competition use case were discussed to delineate the core constituents and processes of the proposed framework. The case of BIM was reviewed. Through the domestic custom luxury yacht design and prototyping student competition, the application of this framework in educational research is demonstrated and the initial quantitative assessment of the framework is carried out. Conclusions: a BIM-based CPLM implementation framework can help the luxury yacht industry capitalize on the global transformation to an information-centric and data-driven new business paradigm in shipbuilding with integrated design, manufacturing and production.
\end{abstract}

Keywords: Building Information Modeling; Luxury Yacht; Communication; Interoperability; Capital; Project Lifecycle Management; Framework

\section{INTRODUCTION}

BIM is developed on the basis of 3D digital design technology, and is usually defined as the digital representation of the physical and functional characteristics of projects [1]. As such, BIM serves as the integrative reservoir of multidisciplinary project data and supports business decision-making through the project's lifecycle [2]. Similar to building facilities, luxury yacht projects were usually delivered in a highly heterogeneous environment that involves broad participation of stakeholders and needs to accommodate a range of key design, engineering, fabrication, construction and operation factors such as cost, time, material, constructability, aesthetic value, performance, and sustainability, to name a few [3]. Recent trends in luxury yacht market also suggest increased client engagement in luxury yacht project lifecycle for informed decision-making and pursuit for greater customization and superior project performance. Market reports [4,5] have shown strong evidence of BIM's business value in enhancing design communication, increasing constructability, streamlining fabrication and installation, and improving overall project outcomes. This paper envisions BIM as a disruptive technology applied in luxury yacht industry. It reviewed the technological premises of BIM and discussed how BIM can shift the paradigm of luxury yacht lifecycle management to embrace a collaborative, integrative and data-intensive project delivery process. The paper proposed and outlined a BIM-based implementation framework for luxury yacht lifecycle management, delineated 
its core constituents and working mechanism, and conducted preliminary qualitative and quantitative evaluation of the proposed framework.

\section{MATERIAL AND METHODS}

\section{PREMISES \& BACKBONE TECHNOLOGY OF BIM}

\section{Parametric modeling}

Designing a luxury yacht design is usually a lengthy, tedious and repetitive process. At the early stage, designers will usually spend months on conceptualization and weighing different options to seek for the optimal design representation that meets the client's expectation. Piles of design sketches are hand-drafted and a tremendous amount of geometric and non-geometric data are generated amid the design evolution. BIM can greatly relieve designers from this labor-intensive workflow and improve design productivity with parametric modeling. Parametric modeling is the foundation to objectbased modeling technology such as BIM [6]. It empowers designers with the capacity to update design parameters, compare design options and visualize the differences simultaneously. Changes in one place will be automatically populated across all affected design, which significantly reduces the work load and eliminates inconsistency among design documents.

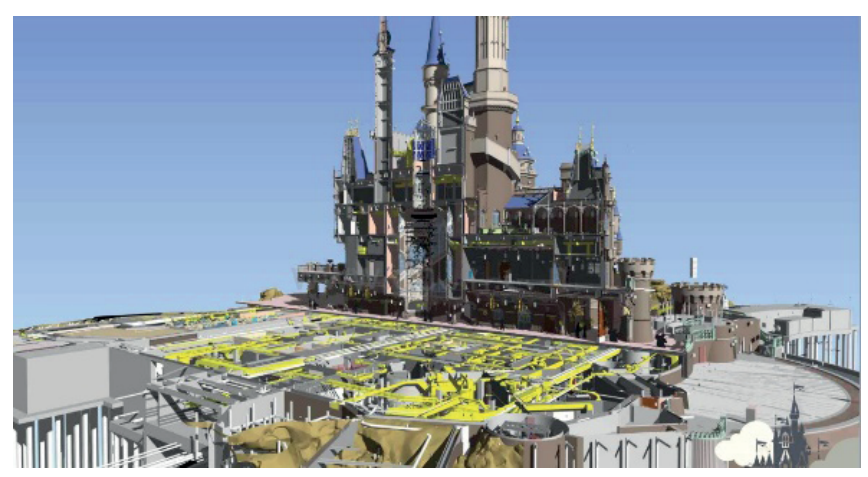

Fig. 1. Revit architecture shaded view of the Enchanted Castle [7]

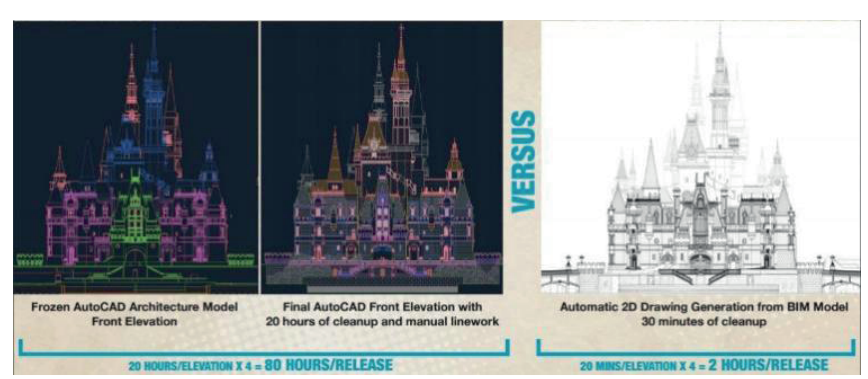

Fig. 2. Documentation generation: BIM vs CAD [7]

BIM software solutions today (e.g. Autodesk Revit, Intergraph Smart 3D) foster intelligent 3D modeling beyond simple geometric forms and shapes. Best practices in BIM implementation have demonstrated the ease of prototyping for designs with non-typical complexities and magnitudes. A superior example can be found in the Shanghai Disney Resort project. As shown in Fig. 1, the Enchanted Storybook Castle married traditional architectural detailing with modern building technology. It presented an extremely complex, dynamic yet harmonious design that meet all the programmatic, operational, accessibility, seismic, fire, mechanical, and technological requirements. Unprecedented $3 \mathrm{D}$ visualization accomplished in the BIM software environment streamlined the communication between designers of 142 disciplines and the client to reach consensus [7]. An exceptional advantage of BIM-based design workflow is the automated documentation generation. Parametric modeling allows customized view representation of the design in literally any standardized or non-standardized manner. Typical building plans, including plan, elevation, section and detail views, can then be produced and updated momentarily. As shown in Fig. 2, by switching to a BIM-based solution, many time-consuming steps could be eliminated from the traditional clean-up process. Instead of manually adjusting each $2 \mathrm{D}$ drawing, drawings were organically produced from the model(s) and modifications would be populated automatically and consistently across all affected sheets. Building standards and specifications can also be integrated within the model environment for further plan proofing and code compliance checking before bid documents are generated.

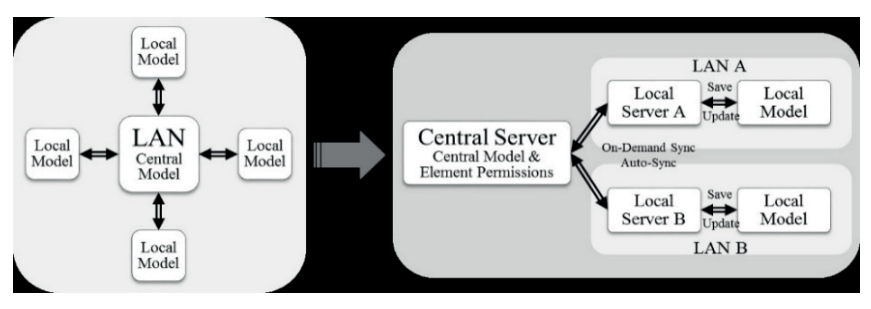

Fig. 3. Server-based file sharing and collaboration [8]

\section{Concurrent collaborative design and construction coordination}

Similar to the AEC industry, there is fragmentation-induced loss of productivity in luxury yacht industry, where projects are typically delivered with highly multidisciplinary, large and complex project teams. BIM provides an opportunity to offer a common information and communication platform and facilitate a truly collaborative working environment [9]. Before the BIM server technology is based on file sharing, to make the work fully synchronized, different teams must be between the model file editing. Full size model synchronization takes considerable time and bandwidth and thus becomes a serious performance and productivity bottleneck to the overall workflow, especially when Wide Area Network (WAN) instead of Local Area Network (LAN) collaboration is desired [8]. Recent advancement in cloud computing and BIM server 
technology offers a much better option to allow componentbased real-time design collaboration and synchronization. Fig. 3 describe the transition to a shared infrastructure. It is expected that future development of collaborative design and construction coordination platform for luxury yacht industry will most likely take the cloud-based BIM server approach.

\section{Information exchange and interoperability standards}

The luxury yacht project lifecycle features largevolume and frequent information generation, exchanging, and updating/reconciliation. The complex engineering and business processes also necessitate the information interdependence among project team members. Highly varied project information with different sizes (e.g. small or large), complexities (structured or unstructured), formats (e.g. text, numeric or graphic) and priorities (low or high) also demand specially tailored, carefully classified exchange protocols. Classification of information exchange protocols not only saves resources, but can also improve quality and efficiency in information exchange process [10]. With a comprehensive underlying database, BIM can readily serve as a common reservoir for capital project lifecycle information. During the design process, designers and domain experts will all contribute to the model authoring. Then quite often, project information will be shared and passed along between team members in various business processes and critical project tasks such as energy simulation and structural analysis. During construction, ad-hoc information generated from day-to-day operation and installation can be quickly fed back to BIM via reality modeling technology such as 3D laser scanning to check against design model information to ensure compliance with performance and quality requirements.

Transferability of BIM-based technology to shipbuilding industry As a new frontier of research, several studies have been conducted to investigate how BIM-enabled technology and best practices could be transferred to the shipbuilding industry. Through structured interviews and literature review on BIM uses in comparison with 3D CAD applications in the shipbuilding industry, four BIM best practices including visualization, clash detection, quantity takeoff and scheduling/4D simulation were identified as highly transferrable [11-13]. Unlike the other three best practices that might find their counterparts in the shipbuilding industry, scheduling/4D simulation was regarded as a unique innovation in the AEC industry by integrating 3D design data with construction schedule data to foster smooth transition of project delivery between the design phase and construction phase. Recent research suggested that data sharing across design sectors and simulation of the construction process to predict time and cost are the key factors for concurrent engineering and integrated lifecycle management in shipbuilding industry [14-16]. Based upon these observations, it is safe to conclude that a BIM-based integrative design and construction approach for shipbuilding project delivery and lifecycle management is not only feasible but highly desirable.

\section{STATE-OF-THE-ART SOFTWARE SOLUTIONS IN LUXURY YACHT INDUSTRY}

Currently, major software vendors in the marine and shipbuilding industry are undergoing significant transformation to embrace intelligent 3D modeling technology and integrated collaborative design, fabrication and production process, which is comparable to the adoption and implementation trajectory of BIM in the AEC industry. The following paragraphs review several leading marine engineering and shipbuilding software applications in the market, and identify the trends and opportunities towards the BIM-based lifecycle management for the luxury yacht industry.

\section{NUPAS-CADMATIC/CADMATIC Marine Design}

Now CADMATIC Marine Design, provides a complete solution for the entire ship and offshore building process with advanced modeling and database technologies that ensures consistent design and production phases. It aims to enable highly efficient global distribution of projects and supports both online and offline sharing of 3D models seamlessly across stakeholders, which achieves full lifecycle management of vessels from early design to operation and decommissioning. Marketed as a highly integrated multidisciplinary solution, CADMATIC Marine Design covers shell, structural, piping and outfitting disciplines. Noticeably, CADMATIC Marine Design is a database-centric intelligent client server system that configures 3D ship models, documents, and component libraries in the primary and replica databases hosted by the database server system. Allegedly, always ensure the integrity of the project data and security, in the CADMATIC distributed design system to obtain the greatest advantage. Thus, in a globally distributed project, it is expected that the data will be updated through the interval set between the remote design sites via an online network such as the Internet, or through a file in an e-mail attachment. Designers can be assured that automated replication systems are responsible for data synchronization, ensuring that design time is not wasted due to incorrect information [17].

\section{Intergraph Smart 3D/Intergraph SmartMarine Enterprise}

Intergraph Smart 3D uses an engineering approach that utilizes real-time parallel design, rules, relationships, and automation [18]. Intergraph Smart 3D provides a range of functions needed to design marine facilities. It is a datacentric, large-scale complex project with centralized visibility and control based on powerful rules and relationships, custom automation capabilities, and integration. Known as the world's first and only next-generation $3 \mathrm{D}$ design solution. The SmartMarine Enterprise, which is comparable to sophisticated BIM applications, provides a full range of design, fabrication, assembly, and lifecycle management capabilities within a single integrated environment [19]. 


\section{TECHNOLOGY TRENDS AND OPPORTUNITIES FOR BIM-BASED LUXURY YACHT CPLM}

Review of the above leading marine and shipbuilding software solutions has helped identify the technology readiness for a paradigm shift in the luxury yacht industry to embrace more collaborative, information-centric and data-intensive business processes. It also seemed to be fairly obvious that Intergraph's SmartMarine 3D resemble advanced BIM applications such as the Autodesk Revit to a great degree. Based upon these observations and evaluations, the paper argues that a luxury yacht is not just a regular product and the scope and complexity of managing a luxury yacht project delivery seem to be more comparable to the capital projects management instead of conventional product management [20]. Therefore, a Capital Project Lifecycle Management (CPLM) approach is proposed by this research instead of the conventional Product Lifecycle Management (PLM).

\section{BIM-based CPLM for luxury yacht industry: implementation framework}

Due to the lack of empirical evidence and established best practices to sufficiently justify the feasibility and validity of the BIM-based CPLM, a generic implementation framework that delineates its rationale and embedded business processes will be of common interest to stakeholders in the luxury yacht industry. The core constituents and processes of the framework are illustrated in Fig. 4, which consists of several interdependent functional layers that channel the project information through the full lifecycle of luxury yacht projects. Reconciling the characteristics of both the AEC and luxury yacht industries, the proposed framework depicts an information-centric lifecycle management approach that organically internalize five functional layers including: the CRM layer, the ERP layer, the Product Information layer, the Process Information layer, and the Network Server Platform layer. Development of this framework embraces and reflects an important principle in technology intervention to product development business process such as BIM's adoption and implementation and market transformation in the AEC industry, which is known as the "people, process and product" (3Ps) rule [21]. Specifically, the CRM layer manages the client relationship and solicits project requirements. The ERP layer manages internal technical and design resources for standardized workflow. Product and Process Information layer represent the information evolution and information stewardship supporting critical business decisions through the design, fabrication, construction and operation of a luxury yacht project. Last but not least, the Network Server Platform layer constitutes the technological infrastructure of the framework, and defines the business protocols for information exchange and sharing among stakeholders and key players in different layers.

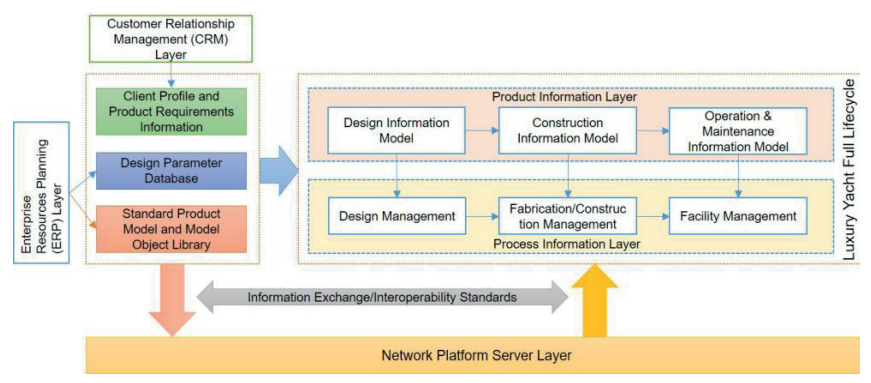

Fig. 4. Proposed implementation framework for full lifecycle luxury yacht design

\section{BIM-based CPLM implementation framework: case study}

As a new concept, there is a lack of empirical evidence and documented literature validating the feasibility of utilizing BIM for capital project lifecycle management in the marine and shipbuilding industry. Other than a few anecdotal claims on promises and business benefits of BIM use in marine and shipbuilding industry, [22] presented an interesting case study of BIM use in new aircraft carrier pier and bulkhead rehabilitation design. Although not about BIM implementation directly involved in the aircraft carrier design and construction, it showcased the decision making and performance of a BIM-facilitated workflow in its waterfront structure design and construction, which provided valuable reference.

Project background: In April 2009, Naval Facilities Engineering Command (NAVFAC) Mid-Atlantic, Norfolk, Virginia, awarded a contract to Moffatt \& Nichol (M\&N)-led MN3M joint venture to design a replacement for Ship Repair Pier 5 at Norfolk Naval Shipyard. The design involved the demolition of two 1,000-foot-long piers (Pier 4 and Pier 5) and five additional buildings to accommodate a new 1,225-footlong, 230-foot-wide pier (Pier 5) supported by more than 1,200 of 36-inch precast, pre-stressed concrete cylinder piles. A primary design goal set by the Navy was to achieve low maintenance needs for the piers' 75-year service life.

Rationale of decision to use BIM: When the design began in 2009, BIM was becoming a standard in vertical structure design, but had hardly been used for piers and other waterfront structures. Traditionally construction documents for waterfront projects relied on 2D drawings with independent manually-created section, plan, and detail views. Bill of materials used for estimation purposes were obtained through traditional quantity takeoff methods, which required an extensive amount of review and coordination between disciplines to identify and mediate element conflicts throughout the construction documents. In this project, which could be very labor intensive using traditional design methods. The Moffatt \& Nichol (M\&N)-led MN3M joint venture decided to utilize BIM to improve coordination among design disciplines, identify potential construction conflicts, reduce errors in design, help visualize the end product, and provide the Navy with a model to be used for asset management. 
Design outcomes and transition to construction: MN3M delivered the final design in February 2010, less than a year after contract award and \$10 million under the Navy's initial estimates for construction. In addition to the design, Moffatt \& Nichol also provides design, planning, analysis, tender and other contract documents, pre-project management and post-project construction services. A comprehensive Autodesk Revit 3D model of the pier and wharf was part of the design deliverables. With the Revit model being produced at $35 \%$ design completion, information exchange and real time collaboration among the MN3M team members were significantly enhanced to allow dynamic detection of conflicts between disciplines and unprecedented visualization of the end design by the Navy. The Revit model was also expected to be used by the Navy as part of their asset management program with great accountability of elements, spaces, equipment and systems in the facility.

\section{BIM-based CPLM implementation framework: use case in academia}

The BIM-based CPLM framework also find its applicability in educational contexts. In 2015 and 2016, students from the South China University of Technology competed in the $3^{\text {rd }}$ and $4^{\text {th }}$ Sunbird (sponsor) National Student Competition on Luxury Yacht Design. The theme of this competition was to design a 100-feet luxury yacht to meet not only technical innovation and functional requirements, but also to integrate client-centered aesthetics, sustainability, cost efficiency and marketability criteria. The deliverables included design drawings, specifications and a physical mockup. This comprehensive competition necessitated interdisciplinary teamwork, collaborative design and construction (mockup) efforts, which offered a great scenario to simulate the BIM-based CPLM implementation framework. A total of fifteen students with diverse backgrounds in fine arts, naval architecture and ocean engineering, industrial engineering and computer science participated in the two competitions.

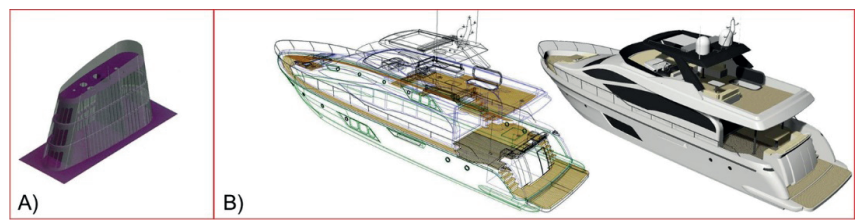

Fig. 5. A) Cabin surface by Smart 3D and B) complex hull surface model by Rhinoceros

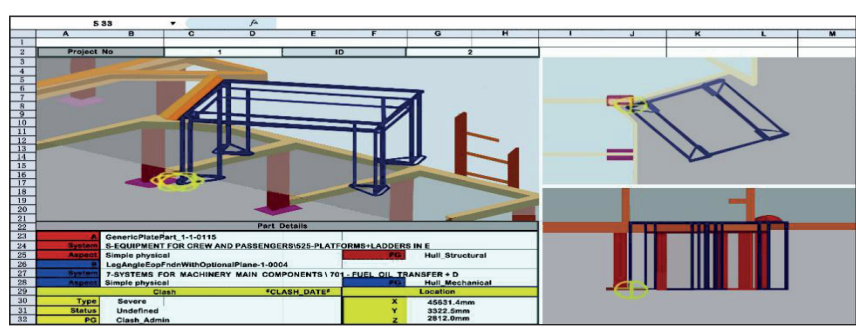

Fig. 6. Material report and clash detection in the hybrid model
Both 3D CAD applications (i.e. AutoCAD and Rhinoceros) and BIM-based solution (i.e. Intergraph Smart 3D) were used to streamline the conceptual design, detail design, construction documentation and mockup construction. Students complimented Smart 3D for its advanced datacentric, rule-driven solution for streamlining the yacht design processes while preserving design data and making it more usable/reusable for documentation generation and constructability review purposes. It is a highly integrative software solution to design and build a luxury yacht. Nevertheless, the disadvantage of Smart 3D is its weakness in complex surface engineering and modeling, for which students relied on Rhinoceros, as shown in Fig. 5. Smart 3D is a mixed model of CAD format that shares engineering data between multiple design teams with SmartPlant Enterprise. Students then utilized the hybrid model to run clash detection for constructability review, and leveraged the rich design data to perform quantity takeoff and generate the Bill of Materials (BOM) for fabrication, procurement and construction purposes (see Fig. 6). Design drawings and specifications were also automated (Fig. 7).
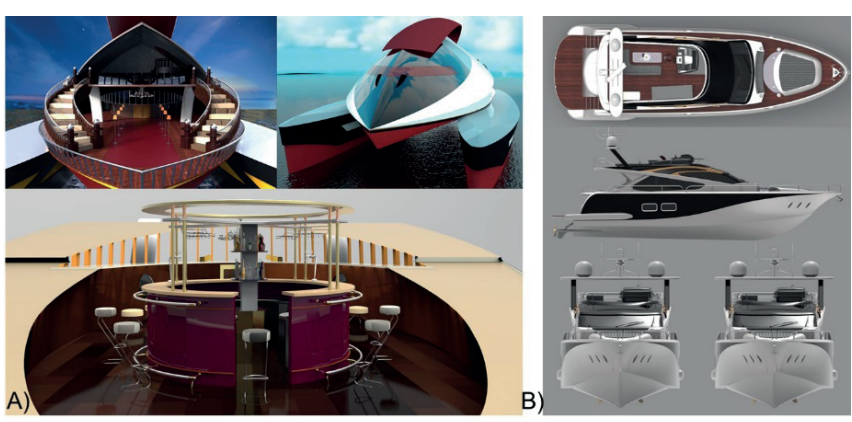

Fig. 7. A) 2015 design renderings and B) 2016 design renderings

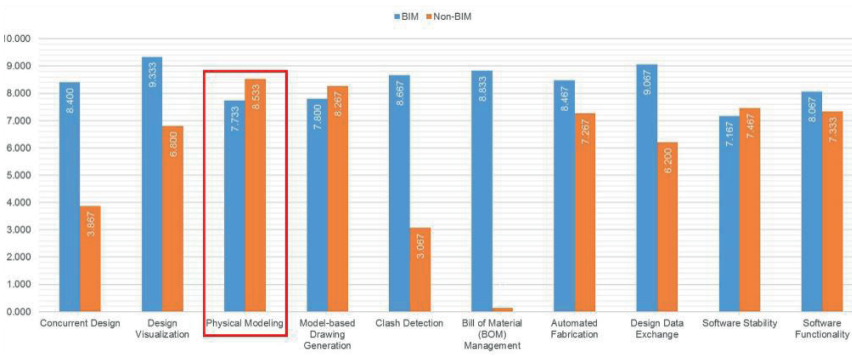

Fig. 8. Preliminary quantitative evaluation of BIM vs. non-BIM ingredients against tenperformance criteria

In the competition debriefing meetings, students reflected that a BIM-facilitated approach could speed up the overall delivery of the project. However, at the conceptual design stage, there was no obvious advantage due to the fact that 3D CADbased solutions were playing more essential roles in geometry design and surface modeling. It was into the detailed design and forward they started to gain significant improvement in efficiency and effectiveness in terms of generating design deliverables and bill of materials, performing clash detection and planning for mockup construction. They also indicated that applications of enterprise resources planning, fabrication 
and construction management systems could greatly improve cost control and reduce overall construction costs. To gain more insights of students' perception towards a BIMintegrated CPLM framework versus traditional luxury yacht project delivery, the 15 students from two competition teams were invited to participate in a short survey that evaluated the BIM-ingredients against the non-BIM ingredients during the competitions, considering that students used both. A set of ten criteria were utilized as assessment measures with a 10-point scale (higher scores indicated more positive perceptions). The results were summarized in Table 1. A two-tailed two-sample unequal-variance student t-test was conducted to calculate if there was a statistically significant difference between students' evaluation on BIM and non-BIM ingredients by each measure. Apparently, except for "Model-based Drawing Generation" and "Software Stability", students' perception towards BIM and non-BIM ingredients differed significantly by the rest of the eight measures. Noticeably, BIM was considered to outperform non-BIM ingredients in seven measures except for "Physical Modeling", which was related to complex surface modeling and was discussed in previous paragraphs (Fig. 8). Therefore, this preliminary quantitative evaluation via an educational use case confirmed that a BIMbased CPLM approach could potentially benefit the luxury yacht industry from various perspectives.

\section{DISCUSSION}

A BIM-facilitated CPLM has the advantage to not only facilitate smooth project delivery via enhanced communication and collaboration among team members, but also leverage consistent project information for decision making throughout the project lifecycle. This is accomplished through a highly integrative common data environment where all project parties can contribute, extract, update, exchange and manage project information with pre-defined business rules and interoperability protocols. With BIM, the project team of the Norfolk Naval Shipyard project was able to eliminate the intrinsic fragmentation in conventional workflow, and proactively involve the owner/operator, designer and contractor establish common project goals and performance requirements. Communication channels enabled by BIM and business intelligence achieved through synthesis of comprehensive multidisciplinary project dallowed the project team to reduce design error, improve constructability, cut project duration and save overall project costs.

\section{CONCLUSIONS}

Information-driven modeling and advanced information technology are transforming business paradigms across different industry sectors, and best practices in one industry may also shed light on the other. This paper investigated opportunities of introducing more collaborative and integrative project delivery and lifecycle management in the luxury yacht industry, based upon principles and best practices of BIM applied in the AEC industry. Literature review suggested strong resemblance of the IT infrastructure and project delivery between the two industries, and capacities of state-of-the-art software applications also demonstrated the readiness for the luxury yacht industry to embrace new business paradigms and BIM-based capital project lifecycle management. An implementation framework was thus proposed to delineate the rationale, constituents and business processes of the BIM-based CPLM. The implementation framework followed the "people, process and product" principle in adopting disruptive technology innovations. The framework was modularized with multiple functional layers that the ultimate goal was to channel both the product and process information of luxury yacht through its full lifecycle. Due to the lack of empirical evidence and best practices, the paper relied on a case study and an educational use case to preliminarily evaluate its feasibility and validity with considerable limitations and possible bias. Nevertheless, the case study and the educational use case consistently confirmed positive perceptions of both professionals and college students towards the BIM-based CPLM in future shipbuilding industry.

As global-distributed design and engineering workflow becoming standard practices, both the AEC industry and shipbuilding industry are facing challenges as well as opportunities in conducting business with data-driven, information-centric decision making. Developing the BIMbased CPLM seems to help enterprises improve business agility and gain competitive advantage in a global market. Future research on BIM-based CPLM can proceed with both macro and micro perspectives, including further development of the implementation framework with comprehensive business process mapping; the common project data environment and data sharing \& exchange protocols; data analytics and big data analytics in sensor-deployed, automated luxury yacht project lifecycle management, to name a few.

\section{ACKNOWLEDGEMENTS}

This paper is supported by the National Natural Science Foundation of China (Grant No.51039006). First of all, I would like to extend my sincere gratitude to my supervisor, Chaohe Chen, for his instructive advice and useful suggestions on my thesis. I am deeply grateful to Dr. Wu for his help in translation studies. I am also deeply grateful of their help in the completion of this thesis. Special thanks should go to the students who have put considerable time and effort into their comments on the questionnaire survey. 
Tab.1. Preliminary quantitative evaluation of BIM vs. non-BIM ingredients in luxury yacht project delivery based upon a survey on student competitions.

\begin{tabular}{|c|c|c|c|c|c|c|c|c|c|c|c|c|c|c|c|c|c|c|c|c|}
\hline \multirow[b]{2}{*}{$\mathrm{N}=15$} & \multicolumn{2}{|c|}{$\begin{array}{c}\text { concurrent } \\
\text { design }\end{array}$} & \multicolumn{2}{|c|}{ visualization } & \multicolumn{2}{|c|}{$\begin{array}{l}\text { physical } \\
\text { modeling }\end{array}$} & \multicolumn{2}{|c|}{$\begin{array}{c}\text { model-based } \\
\text { drawing }\end{array}$} & \multicolumn{2}{|c|}{$\begin{array}{c}\text { clash } \\
\text { detection }\end{array}$} & \multicolumn{2}{|c|}{$\begin{array}{c}\text { bill of } \\
\text { material }\end{array}$} & \multicolumn{2}{|c|}{$\begin{array}{l}\text { automated } \\
\text { fabrication }\end{array}$} & \multicolumn{2}{|c|}{$\begin{array}{l}\text { design data } \\
\text { exchange }\end{array}$} & \multicolumn{2}{|c|}{$\begin{array}{l}\text { software } \\
\text { stability }\end{array}$} & \multicolumn{2}{|c|}{$\begin{array}{c}\text { software } \\
\text { functionality }\end{array}$} \\
\hline & BIM & no & BIM & no & BIM & no & BIM & no & BIM & no & BIM & no & BIM & no & BIM & no & BIM & no & BIM & no \\
\hline 1 & 8 & 4 & 9 & 7 & 8 & 8 & 8 & 7 & 8 & 3 & 7 & 0 & 9 & 7 & 9 & 6 & 7 & 7 & 8 & 7 \\
\hline 2 & 9 & 4 & 8 & 5 & 9 & 9 & 8 & 7 & 8 & 3 & 9 & 2 & 9 & 8 & 9 & 7 & 7 & 8 & 8 & 7 \\
\hline 3 & 9 & 4 & 8 & 7 & 9 & 8 & 8 & 8 & 8 & 3 & 9 & 0 & 8 & 7 & 9 & 6 & 8 & 7 & 8 & 8 \\
\hline 4 & 8 & 5 & 8 & 7 & 8 & 6 & 9 & 8 & 8 & 3 & 9 & 0 & 8 & 8 & 9 & 6 & 7 & 8 & 9 & 7 \\
\hline 5 & 8 & 4 & 10 & 6 & 7 & 9 & 8 & 8 & 9 & 2 & 9 & 0 & 7 & 6 & 8 & 6 & 7 & 7 & 8 & 8 \\
\hline 6 & 8 & 5 & 10 & 8 & 7 & 9 & 7 & 9 & 9 & 2 & 9 & 0 & 9 & 6 & 8 & 6 & 7 & 7 & 9 & 7 \\
\hline 7 & 10 & 4 & 10 & 7 & 8 & 9 & 8 & 9 & 9 & 3 & 7 & 0 & 9 & 7 & 8 & 6 & 8 & 8 & 8 & 8 \\
\hline 8 & 8 & 6 & 9 & 6 & 7 & 9 & 7 & 8 & 8 & 5 & 9 & 0 & 9 & 7 & 9 & 5 & 7 & 7 & 8 & 7 \\
\hline 9 & 7 & 4 & 10 & 7 & 7 & 9 & 8 & 9 & 9 & 3 & 9 & 0 & 9 & 8 & 10 & 7 & 7 & 7 & 8 & 8 \\
\hline 10 & 9 & 3 & 10 & 6 & 7 & 9 & 8 & 9 & 8 & 5 & 10 & 0 & 8 & 7 & 10 & 6 & 7 & 7 & 8 & 7 \\
\hline 11 & 8 & 4 & 10 & 7 & 8 & 9 & 7 & 9 & 10 & 2 & 10 & 0 & 8 & 8 & 10 & 6 & 7 & 7 & 8 & 7 \\
\hline 12 & 9 & 2 & 10 & 7 & 8 & 8 & 8 & 8 & 10 & 3 & 10 & 0 & 8 & 7 & 9 & 6 & 7 & 8 & 8 & 7 \\
\hline 13 & 9 & 1 & 10 & 7 & 8 & 8 & 8 & 7 & 10 & 1 & 9 & 0 & 9 & 9 & 10 & 7 & 7 & 8 & 8 & 7 \\
\hline 14 & 8 & 4 & 9 & 8 & 8 & 9 & 8 & 9 & 8 & 5 & 7 & 0 & 9 & 7 & 9 & 6 & 7 & 8 & 8 & 7 \\
\hline 15 & 8 & 4 & 9 & 7 & 7 & 9 & 7 & 9 & 8 & 3 & 9 & 0 & 8 & 7 & 9 & 7 & 7 & 8 & 8 & 8 \\
\hline Mean & 8.40 & 3.87 & 9.33 & 6.80 & 7.73 & 8.53 & 7.80 & 8.27 & 8.67 & 3.07 & 8.83 & 0.13 & 8.47 & 7.27 & 9.07 & 6.20 & 7.17 & 7.47 & 8.07 & 7.33 \\
\hline median & 8 & 4 & 10 & 7 & 8 & 9 & 8 & 8 & 8 & 3 & 9 & 0 & 9 & 7 & 9 & 6 & 7 & 7 & 8 & 7 \\
\hline variance & 0.54 & 1.41 & 0.67 & 0.60 & 0.47 & 0.70 & 0.31 & 0.64 & 0.67 & 1.35 & 1.06 & 0.27 & 0.41 & 0.64 & 0.50 & 0.31 & 0.13 & 0.27 & 0.21 & 0.24 \\
\hline $\mathrm{SD}$ & 0.74 & 1.19 & 0.82 & 0.78 & 0.70 & 0.83 & 0.56 & 0.80 & 0.82 & 1.16 & 1.03 & 0.52 & 0.64 & 0.80 & 0.70 & 0.56 & 0.36 & 0.52 & 0.46 & 0.49 \\
\hline $\mathrm{t}$-Test & \multicolumn{2}{|c|}{0.000} & \multicolumn{2}{|c|}{0.000} & \multicolumn{2}{|c|}{0.008} & \multicolumn{2}{|c|}{0.076} & \multicolumn{2}{|c|}{0.000} & \multicolumn{2}{|c|}{0.000} & \multicolumn{2}{|c|}{0.000} & \multicolumn{2}{|c|}{0.000} & \multicolumn{2}{|c|}{0.077} & \multicolumn{2}{|c|}{0.000} \\
\hline Statistically & \multicolumn{2}{|c|}{ Yes } & \multicolumn{2}{|c|}{ Yes } & \multicolumn{2}{|c|}{ Yes } & \multicolumn{2}{|c|}{ No } & \multicolumn{2}{|c|}{ Yes } & \multicolumn{2}{|c|}{ Yes } & & & & & & & & \\
\hline
\end{tabular}

\section{REFERENCES}

1. NIBS. 2007. Unite States National Building Information Modeling Standard, Version 1 - Part 1: Overview, Principles, and Methodologies Washington, DC.

2. J. Yang, X. Zhang, 2009. Building Information Integration Model and Supporting Technology Standard. Construction Technology, 38(10), 109-112.

3. W. Wu, R. R. A. Issa, 2010. Feasibility of integrating building information modeling and LEED certification process. In: International Conference on Computing in Civil and Building Engineering. Nottingham. pp. 161.

4. McGraw-Hill Construction, 2009. The Business Value of BIM: Getting Building Information Modeling to the Bottom Line. M.-H. Construction, Bedford, MA.

5. McGraw-Hill Construction. 2012. The Business Value of BIM in North America: Multi-Year Trend Analysis and User Ratings (2007-2012). M.-H. Construction, Bedford, MA.

6. C. Eastman, P. Teicholz, R. Sacks, K. Liston, 2011. BIM Handbook: A Guide to Building Information Modeling for Owners, Managers, Designers, Engineers and Contractors (2nd ed.). NJ: John Wiley and Sons, Hoboken.
7. AIA, 2014. AIA TAP BIM Awards - 2014 Professionas' Choice BIM Award. http://bimforum.org/wp-content/ uploads/2014/04/9ab-2014-Professionals-Choice-BIMAward-MASTER-FINAL-PPT-04172014. pdf.

8. W. Wu, R. R. A. Issa, 2012. Leveraging Cloud-BIM for LEED Automation. Journal of Information Technology in Construction, 17, 367-384.

9. B. Becerik-Gerber, K. Ku, F. Jazizadeh, 2012. BIM-Enabled Virtual and Collaborative Construction Engineering and Management. Journal of Professional Issues in Engineering Education and Practice, 138(3), pp. 234-245.

10. W. Zhong, T. Jiang, 2014. Collaborative Construction based on BIM Standard. Journal of Information Technology in Civil Engineering and Architecture, 6(5), pp. 95-101.

11. L. Ran, 2015. Transferring best practices enabled by Building information modeling (BIM) in Architecture, Engineering and Construction (AEC) to shipbuilding industry: An explorative study. (Industry Engineering and Management Master), Aalto University, Espoo, Finland. https://aaltodoc. aalto.fi/bitstream/handle/123456789/17703/master_Ran_ Luming_2015.pdf?sequence $=1$.

12. L. Ran, V. Singh, 2016a. Building information modellingenabled best practices in AEC and takeaways for Finnish 
shipbuilding industry. International Journal of Product Lifecycle Management, 9(3), pp. 238.

13. L. Ran, V. Singh, 2016b. Comparing BIM in Construction with 3D Modeling in Shipbuilding Industries: Is the Grass Greener on the Other Side? In A. Bouras, B. Eynard, S. Foufou, \& K.-D. Thoben (Eds.), Product Lifecycle Management in the Era of Internet of Things (pp. 193202): Springer International Publishing.

14. M. Braglia, D. Castellano, M. Frosolini, 2014. Computeraided activity planning (CAAP) in large-scale projects with an application in the yachting industry. Computers in Industry, 65(4), pp. 733-745.

15. K. Hiekata, M. Grau, 2014. Case Studies for Concurrent Engineering Concept in Shipbuilding Industry. In J. Cha, S.-Y. Chou, J. Stjepandić, R. Curran, \& W. Xu (Eds.), Moving Integrated Product Development to Service Clouds in the Global Economy (Vol. 1, pp. 102-111): IOS Press Inc.

16. W. Tann, H.-J. Shaw, R. Bronsart, 2005. Integrating the Collaborative Environment in Shipbuilding: An Implementation Strategy. Journal of Ship Production, 21(1), pp. 37-45.

17. CADMATIC, 2016. CADMATIC Marine Design. http:// www.nupas-cadmatic.com/company/overview.html.

18. Intergraph, 2016a. Intergraph Smart 3D. http:// ppm.intergraph.com/products/3d-product-family/ intergraph-smart-3d.

19. Intergraph, 2016b. SmartMarine Enterprise: Improving the Complete Marine Life Cycle. http://viewer.zmags.com/ publication/f19dd27d\#/f19dd27d/1.

20. Intergraph, 2009. Capital Project Life Cycle Management (cPLM) vs. Product Life Cycle Management (PLM) for the Shipbuilding, Marine, and Offshore Industries. http://www.intergraph.com/assets/pdf/ IntergraphcPLMvsPLMwhitepaper.pdf.

21. N. Gu, K. London, 2010. Understanding and facilitating BIM adoption in the AEC industry. Automation in Construction, 19(8), pp. 988-999.

22. J. E. Gaul, M. N. Rieger, 2016. BIM Use in New Aircraft Carrier Pier and Bulkhead Rehabilitation Design. http://www.aecbytes.com/feature/2016/BIMPierBulkheadDesign.html.

\section{CONTACT WITH THE AUTHORS}

\section{Wei Wu}

Department of Construction Management California State University at Fresno Fresno, CA

USA 